\title{
Erratum to: Cancer in childhood, adolescence, and young adults: a population-based study of changes in risk of cancer death during four decades in Norway
}

\author{
Sara Ghaderi • Rolv Terje Lie · Dag Moster • \\ Ellen Ruud • Astri Syse · Finn Wesenberg • \\ Tone Bjørge
}

Published online: 12 October 2013

(C) Springer Science+Business Media Dordrecht 2013

Erratum to: Cancer Causes Control (2012)

23:1297-1305

DOI 10.1007/s10552-012-0007-x

During the work on our project, a small programming error was discovered. A small population of children who were not born in Norway, especially during the first 2 years of the study period (1965-1966) was included in the original manuscript.
The online version of the original article can be found under doi:10.1007/s10552-012-0007-x.

S. Ghaderi $(\bowtie) \cdot$ R. T. Lie $\cdot$ D. Moster · T. Bjørge Department of Public Health and Primary Health Care, University of Bergen, Kalfarveien 31, 5018 Bergen, Norway e-mail: Sara.Ghaderi@isf.uib.no

R. T. Lie · T. Bjørge

Medical Birth Registry of Norway, Norwegian Institute of Public Health, Bergen, Norway

\section{Moster}

Department of Clinical Medicine, University of Bergen, Bergen, Norway
All analyses of the published paper were repeated. Fortunately, only small differences in numbers were observed, and all conclusions remained unchanged. The revised Tables 1, 2, 3 and Figs. 1, 2 are presented below.

The authors apologize for any inconvenience this may have caused.

\author{
D. Moster \\ Department of Pediatrics, Haukeland University Hospital, \\ Bergen, Norway

\section{E. Ruud · F. Wesenberg} \\ Section for Pediatric Hematology and Oncology, Women and \\ Children's Division, Department of Pediatrics Medicine, Oslo \\ University Hospital, Oslo, Norway
}

A. Syse

Department of Research, Cancer Registry of Norway, Oslo, Norway 
Table 1 Characteristics of the cancer patients born in Norway during 1965-1985
${ }^{a}$ Death cases within 5 years after diagnosis

b $83.8 \%$ were Wilms tumor cases

c This site includes malignant neoplasm of the thyroid gland $(44.6 \%)$, suprarenal gland $(16.8 \%)$, pituitary gland $(20.8 \%)$, pineal gland $(8.1 \%)$, craniopharyngeal canal $(9.2 \%)$, and others $(0.6 \%)$

${ }^{\mathrm{d}}$ Forty-eight patients who died before 1969 lack cause of death specification

\begin{tabular}{|c|c|c|c|}
\hline & $\begin{array}{l}\text { No. of } \\
\text { individuals (\%) }\end{array}$ & $\begin{array}{l}\text { Total no. } \\
\text { of deaths }{ }^{\mathrm{a}}(\%)\end{array}$ & $\begin{array}{l}\text { No. of cancer } \\
\text { deaths }(\%)\end{array}$ \\
\hline \multicolumn{4}{|l|}{ Gender } \\
\hline Male & $3,268(56.3)$ & 889 (59.9) & $846(60.0)$ \\
\hline Female & $2,534(43.7)$ & $601(40.1)$ & $563(40.0)$ \\
\hline \multicolumn{4}{|l|}{ Year of birth } \\
\hline 1965-1969 & $1,504(25.9)$ & $524(35.0)$ & $465(33.0)$ \\
\hline 1970-1974 & $1,456(25.1)$ & $413(27.6)$ & 403 (28.6) \\
\hline 1975-1979 & $1,306(22.5)$ & $276(18.4)$ & $264(18.7)$ \\
\hline $1980-1985$ & $1,536(26.5)$ & $286(19.1)$ & $277(19.7)$ \\
\hline \multicolumn{4}{|l|}{ Age at diagnosis } \\
\hline $0-14$ & $2,481(42.8)$ & $1,007(67.2)$ & $938(66.6)$ \\
\hline $15-19$ & $1,289(22.2)$ & $257(17.1)$ & $249(17.7)$ \\
\hline $20-24$ & $2,032(35.0)$ & $235(15.7)$ & $222(15.8)$ \\
\hline \multicolumn{4}{|l|}{ Year of diagnosis } \\
\hline 1965-1974 & $534(9.2)$ & $349(23.3)$ & $297(21.1)$ \\
\hline 1975-1984 & $1,230(21.2)$ & $474(31.6)$ & $462(32.8)$ \\
\hline 1985-1994 & $2,128(36.7)$ & $441(29.4)$ & $423(30.0)$ \\
\hline 1995-2004 & $1,639(28.2)$ & 219 (14.6) & $211(15.0)$ \\
\hline 2005-2009 & $271(4.7)$ & $16(1.1)$ & $16(1.1)$ \\
\hline \multicolumn{4}{|l|}{ Cancer site (ICD-7 codes) } \\
\hline Kidney $(180.0)^{\mathrm{b}}$ & $154(2.7)$ & $43(2.9)$ & $36(2.6)$ \\
\hline Eye (192) & $98(1.7)$ & $7(0.5)$ & $6(0.4)$ \\
\hline CNS tumor (193) & $1,192(20.5)$ & $407(27.2)$ & $385(27.3)$ \\
\hline Thyroid gland and other endocrine glands $(194-5)^{\mathrm{c}}$ & $370(6.4)$ & 57 (3.8) & $53(3.8)$ \\
\hline Bone and connective tissue (196-7) & $393(6.8)$ & $156(10.4)$ & $154(10.9)$ \\
\hline Lymphatic system (206) & $742(12.8)$ & $133(9)$ & $125(8.9)$ \\
\hline Hodgkin lymphoma & $414(55.8)$ & $23(17.3)$ & $20(16.0)$ \\
\hline Non-Hodgkin lymphoma & $270(36.4)$ & $92(69.2)$ & $88(70.4)$ \\
\hline Other & $58(7.8)$ & $18(13.5)$ & $17(13.6)$ \\
\hline Hematopoietic system (207) & $1,008(17.4)$ & $479(32.0)$ & $454(32.2)$ \\
\hline Acute lymphatic leukemia (ALL) & $625(61.9)$ & $217(45.3)$ & $213(46.9)$ \\
\hline Acute myelogenic leukemia (AML) & $191(18.9)$ & $127(26.5)$ & $121(26.7)$ \\
\hline Other & $194(19.2)$ & $135(28.2)$ & $120(26.4)$ \\
\hline Cervix uteri (171) & $68(1.2)$ & $10(0.7)$ & $10(0.7)$ \\
\hline Ovary (175) & $100(1.7)$ & $12(0.8)$ & $12(0.9)$ \\
\hline Testis (178) & $741(12.8)$ & $45(3.0)$ & $40(2.8)$ \\
\hline Melanoma (190) & $471(8.1)$ & $20(1.3)$ & $18(1.3)$ \\
\hline Other & $465(8.0)$ & $130(8.7)$ & $116(8.2)$ \\
\hline \multicolumn{4}{|l|}{ Year of death } \\
\hline $1969-1974^{\mathrm{d}}$ & & 299 (19.9) & 248 (17.6) \\
\hline 1975-1984 & & $454(30.3)$ & $442(31.4)$ \\
\hline 1985-1994 & & $450(30.0)$ & $437(31.0)$ \\
\hline 1995-2004 & & $261(17.4)$ & 248 (17.6) \\
\hline 2005-2008 & & $35(2.3)$ & $34(2.4)$ \\
\hline Total & $5,802(100.0)$ & 1,499 (100.0) & $1,409(100.0)$ \\
\hline
\end{tabular}


Table 2 Hazard ratios (HRs) of death among the cancer patients diagnosed before age 25 relative to individuals without cancer by year of diagnosis, with $95 \%$ confidence intervals (CI) estimated by a timedependent Cox regression model ${ }^{\mathrm{a}}$

\begin{tabular}{lll}
\hline & $\begin{array}{l}\text { 5-year follow-up } \\
\text { after diagnosis } \\
\left(\mathrm{n}^{\mathrm{c}}=1,499 / 5,802\right)\end{array}$ & $\begin{array}{l}\text { Total follow-up } \\
(\mathrm{n}=1,793 / 5,802)\end{array}$ \\
\hline Year of diagnosis $^{\mathrm{d}}$ & & \\
$1965-1974$ & $361.5(313.9,416.2)$ & $79.7(69.8,91.0)$ \\
$1975-1984$ & $245.2(216.8,277.4)$ & $46.2(41.3,51.5)$ \\
$1985-1994$ & $94.1(83.4,106.1)$ & $28.9(26.1,32.1)$ \\
$1995-2004$ & $41.3(34.8,49.1)$ & $26.0(22.1,30.5)$ \\
$2005-2009$ & $19.3(9.2,40.7)$ & $25.4(12.1,53.4)$ \\
\hline
\end{tabular}

${ }^{a}$ The analysis was adjusted for gender and year of birth (1965-1969, 1970-1974, 1975-1979, and 1980-1985)

b The follow-up time was from birth until 5 years after diagnosis

${ }^{c} \mathrm{~N}$ represents the number of deaths/total number of cancer patients

d The analyses were performed relative to the general population
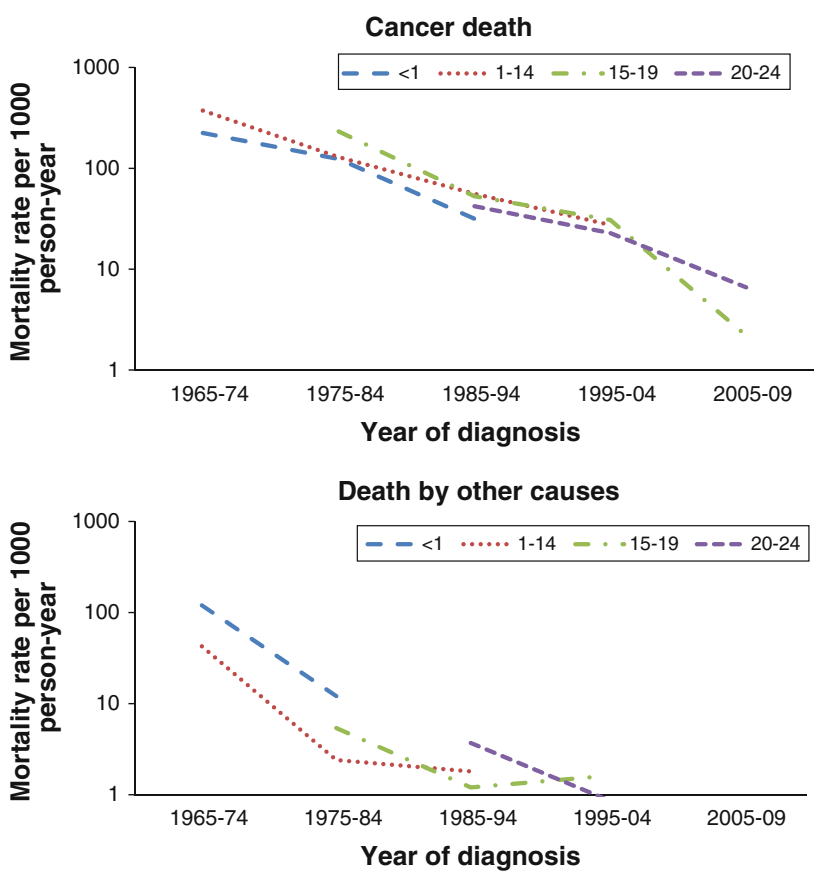

Fig. 1 Early death rates (5 years after diagnosis) caused by cancer and other causes among cancer patients by age at diagnosis $(<1,1-14$, $15-19$, and 20-24 years) per 1,000 person-years. Note that patients diagnosed in the period 2005-2009 were at least 20 years of age at the time of diagnosis. The y-axes are presented in logarithmic scale

Table 3 Sub-hazard ratios (SHRs) with $95 \%$ confidence intervals (CI) for early cancer death (5 years after diagnosis) estimated in a multivariate competing risk model for cancer patients diagnosed before the age of 25

\begin{tabular}{|c|c|c|c|c|c|c|c|}
\hline & $\begin{array}{l}\text { CNS tumors }\left(193^{\mathrm{a}}\right) \\
\left(\mathrm{n}^{\mathrm{b}}=383 / 1192\right)\end{array}$ & $\begin{array}{l}\text { Bone and } \\
\text { connective } \\
\text { tissue }(196-7) \\
(\mathrm{n}=154 / 393)\end{array}$ & $\begin{array}{l}\text { Lymphatic } \\
\text { system } \\
(206) \\
(\mathrm{n}=125 / 742)\end{array}$ & $\begin{array}{l}\text { Hematopoietic } \\
\text { system: all } \\
\text { combined }(207) \\
(\mathrm{n}=453 / 1,008)\end{array}$ & $\begin{array}{l}\text { Acute } \\
\text { lymphatic } \\
\text { leukemia } \\
(\text { ALL) } \\
(\mathrm{n}=212 / 625)\end{array}$ & $\begin{array}{l}\text { Acute } \\
\text { myelogenic } \\
\text { leukemia (AML) } \\
(\mathrm{n}=121 / 191)\end{array}$ & $\begin{array}{l}\text { All cancer } \\
\text { sites } \\
(\mathrm{n}=1,409 / 5,802)\end{array}$ \\
\hline \multicolumn{8}{|l|}{ Gender } \\
\hline Male & 1 & 1 & 1 & 1 & 1 & 1 & 1 \\
\hline Female & $0.82(0.66,1.01)$ & $0.92(0.67,1.27)$ & $0.81(0.56,1.18)$ & $0.86(0.71,1.03)$ & $0.91(0.69,1.22)$ & $0.63(0.43,0.91)$ & $0.83(0.75,0.93)$ \\
\hline \multicolumn{8}{|c|}{ Year of diagnosis } \\
\hline 1965-1974 & 1 & 1 & 1 & 1 & 1 & 1 & 1 \\
\hline 1975-1984 & $0.69(0.53,0.92)$ & $0.65(0.33,1.28)$ & $0.58(0.27,1.27)$ & $0.43(0.34,0.54)$ & $0.45(0.32,0.64)$ & $0.58(0.32,1.07)$ & $0.60(0.52,0.70)$ \\
\hline 1985-1994 & $0.41(0.30,0.55)$ & $0.41(0.20,0.82)$ & $0.28(0.12,0.65)$ & $0.31(0.23,0.41)$ & $0.32(0.21,0.48)$ & $0.77(0.38,1.38)$ & $0.38(0.32,0.45)$ \\
\hline 1995-2004 & $0.25(0.16,0.39)$ & $0.33(0.16,0.71)$ & $0.17(0.07,0.40)$ & $0.29(0.20,0.43)$ & $0.30(0.17,0.53)$ & $0.48(0.22,1.06)$ & $0.28(0.23,0.35)$ \\
\hline 2005-2009 & $0.05(0.01,0.23)$ & $0.11(0.01,0.93)$ & c & $0.10(0.02,0.42)$ & $0.18(0.02,1.66)$ & $0.22(0.03,1.46)$ & $0.15(0.09,0.26)$ \\
\hline \multicolumn{8}{|c|}{ Age at diagnosis } \\
\hline $0-14$ & 1 & 1 & 1 & 1 & 1 & 1 & 1 \\
\hline $15-19$ & $0.69(0.49,0.97)$ & $0.93(0.65,1.34)$ & $0.96(0.59,1.57)$ & $2.16(1.56,2.89)$ & $2.74(1.75,4.28)$ & $1.36(0.81,2.26)$ & $0.73(0.62,0.86)$ \\
\hline $20-24$ & $0.88(0.60,1.30)$ & $0.66(0.37,1.18)$ & $0.52(0.29,0.93)$ & $1.92(1.29,2.87)$ & $2.21(1.18,4.13)$ & $0.93(0.53,1.64)$ & $0.46(0.38,0.56)$ \\
\hline
\end{tabular}

a ICD-7 codes

${ }^{b} \mathrm{~N}$ represents the number of cancer deaths/total number of cancer patients

${ }^{\mathrm{c}}$ There were no death cases 

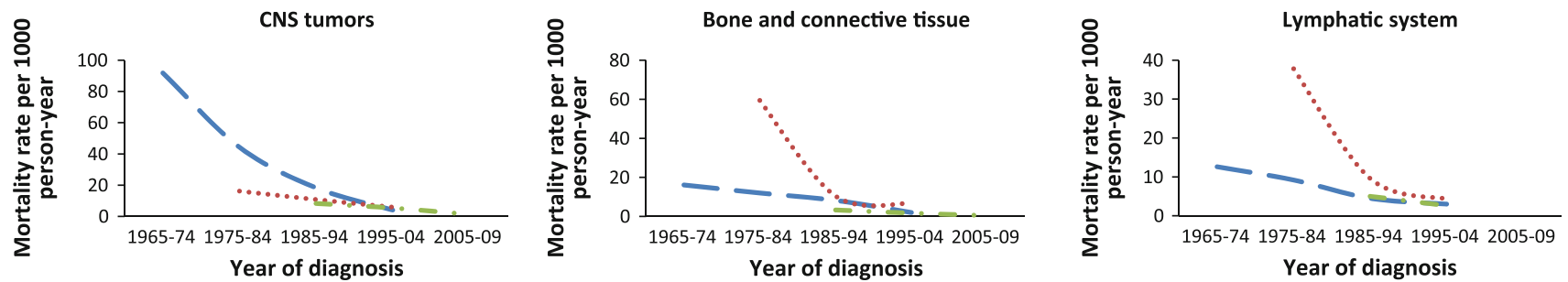

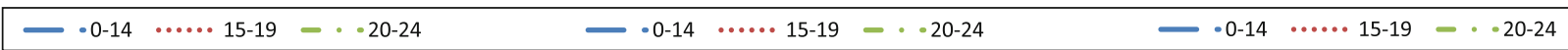
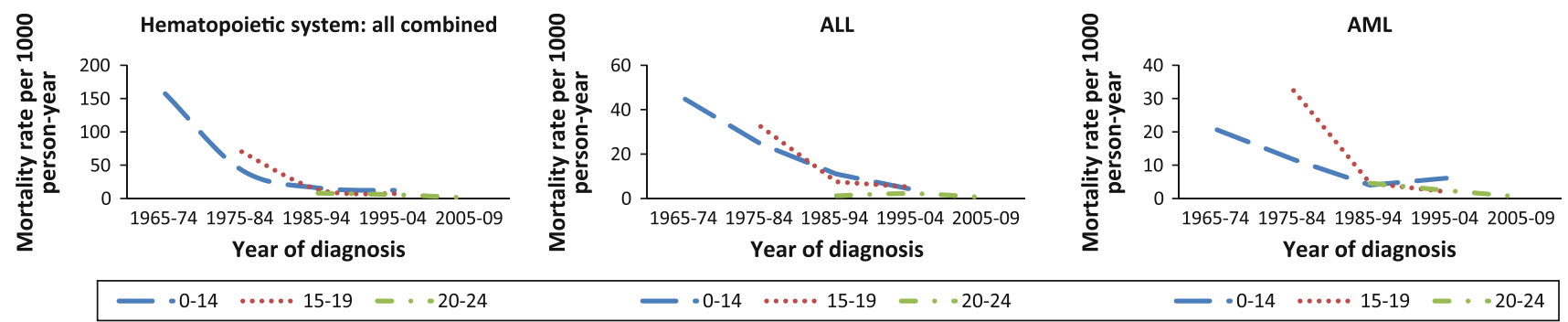

Fig. 2 Cause-specific early death rate (5 years after diagnosis) among cancer patients by age at diagnosis (0-14, 15-19, and 20-24 years) per 1,000 person-years for four major cancer sites (including ALL and AML). Note that patients diagnosed in the period 2005-2009 were at least 20 years of age at the time of diagnosis. $A L L$ acute lymphatic leukemia, $A M L$ acute myelogenic leukemia 